\title{
Zinc Gluconate
}

National Cancer Institute

\section{Source}

National Cancer Institute. Zinc Gluconate. NCI Thesaurus. Code C84248.

A nutritional supplement containing the zinc salt form of gluconic acid for the purpose of providing zinc. As an essential trace element, zinc is of key importance in many biological processes, acts as an antioxidant and strengthens the immune system. Although the mechanism of action is not completely known, zinc supplementation may be used to increase immunity against viruses or may interfere with the replication of certain viruses, such as the human papillomavirus (HPV). 